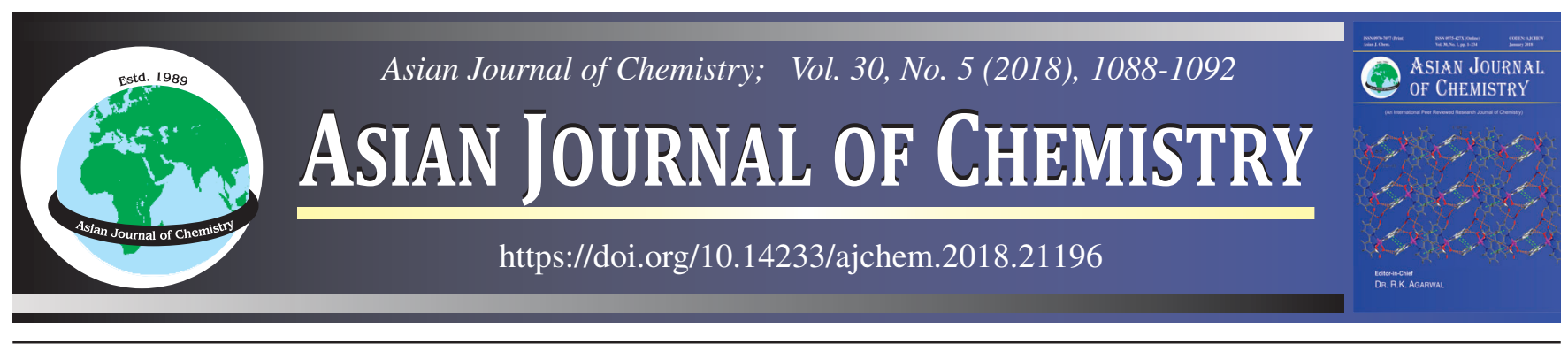

\title{
Development and Validation of 2D GC-FID Method for Quantitative Analysis of cis- and trans-Hexyl Cinnamic Aldehyde and its Major Impurity 2-Hexyl-2-decenal
}

\author{
Chandrakant V. Zure and Rahul V. Pinjari*
}

School of Chemical Sciences, Swami Ramanand Teerth Marathwada University, Nanded-431 606, India

*Corresponding author: E-mail: rvp.chem@srtmun.ac.in

Received: 23 December 2017;

Accepted: 1 February 2018;

Published online: 29 March 2018;

AJC-18846

Hexyl cinnamic aldehyde (HCA) is an aroma chemical used as a flavour and fragrance ingredient, which is synthesized in huge quantity worldwide. The hexyl cinnamic aldehyde present in the trans- and cis-forms with nearly 95:5 ratio. The 2-hexyl-dec-2-enal (HDEA) is process impurity in hexyl cinnamic aldehyde, which may amount upto 2.0\%. However use of 2-hexyl-dec-2-enal is prohibited in the fragrance, while in flavours the Flavour and Extract Manufacturer Association (FEMA) has permitted its concentration only up to 5.0 ppm. Thus a simple and rapid two dimensional gas chromatographic method (2D-GC) for quantitative analysis of (cis-/trans-)hexyl cinnamic aldehyde and 2-hexyl-dec-2-enal have been developed. The non-polar and polar capillary columns are connected in series and the flame ionization detector with nitrogen carrier is used. The method yields a well separated cis-hexyl cinnamic aldehyde, trans-hexyl cinnamic aldehyde and 2-hexyl-dec-2-enal peaks from the other impurities. The method is validated according to the ICH guideline for precision, recovery, linearity, and limit of detection.

Keywords: Two dimensional gas chromatography, Hexyl cinnamic aldehyde, 2-Hexyl dec-2-enal.

\section{INTRODUCTION}

Hexyl cinnamic aldehyde (HCA), generically known as 2-hexyl-3-phenyl-2-propenal (Table-1) is an aroma chemical. The chamomile flower is one of the natural sources of HCA [1]. It is synthesized primarily from aldol condensation reaction between benzaldehyde and octanal [2]. This yields a HCA in the trans- and cis-forms with nearly 95:5 ratio. These isomers of HCA may differ in characteristic odor. The concentration of HCA in flavour and fragrance compound in different consumer products ranges from 0.7 to $17.1 \%$. It is unrestrictedly used in non-skin contact products like candles, air fresheners and room sprays [3]. It is also used in chemical, pharmaceutical and petroleum industries as precursor. The structure of HCA and the process impurities present in it are shown in Table-1. The 2-hexyl-dec-2-enal (HDEA) is a major impurity amounting upto $\sim 2.0 \%(\mathrm{w} / \mathrm{w})$. However, its use is prohibited in the fragrance, while as per flavour and extract manufacturer association (FEMA) amount of HDEA allowed in flavour is upto $5.0 \mathrm{ppm}$ [4]. The presence of HDEA in higher concentration may adversely influence the quality of HCA [5], which makes it essential to control and analyze the HDEA precisely. The other impurities like 2-pentyl-3-phenyl-2-propenal, benzyl alcohol, and octanol may also present in HCA (Table-1). The separation of the cis-HCA, trans-HCA and HDEA from other impurities namely 2-pentyl-3-phenyl-2-propenal, benzyl alcohol, octanol and benzyl benzoate have not been reported yet. These impurities excluding HDEA and cis-HCA as well as trans-HCA can be separated on non-polar stationary phase; however resolution of the HDEA is achieved only on polar columns [6].

Thus in this paper, the accurate, fast, precise and reliable method is developed for separation of HDEA and cis- as well as trans-isomers of HCA in a single run by employing two dimensional gas chromatography (2D-GC) [7], where the nonpolar and polar columns are used in series. The method is further validated according to $\mathrm{ICH}$ guidelines. The validation helps to builds a degree of confidence, not only for developer but also for users [8].

\section{EXPERIMENTAL}

Standard sample and test sample of hexyl cinnamic aldehyde (HCA) were received from Eternis Fine chemicals Limited, Kurkumbh, Pune. The analytical grade acetone as well as methanol and HPLC grade isopropyl alcohol were purchased from Merck, whereas octanaol, 2-pentyl-3-phenyl2-propenal, 2-hexyl-dec-2-enal (HDEA), benzyl alcohol, etc. were purchased from sigma Aldrich. The Shimadzu Analytical balance, Agilent 7890B gas chromatography with flame 


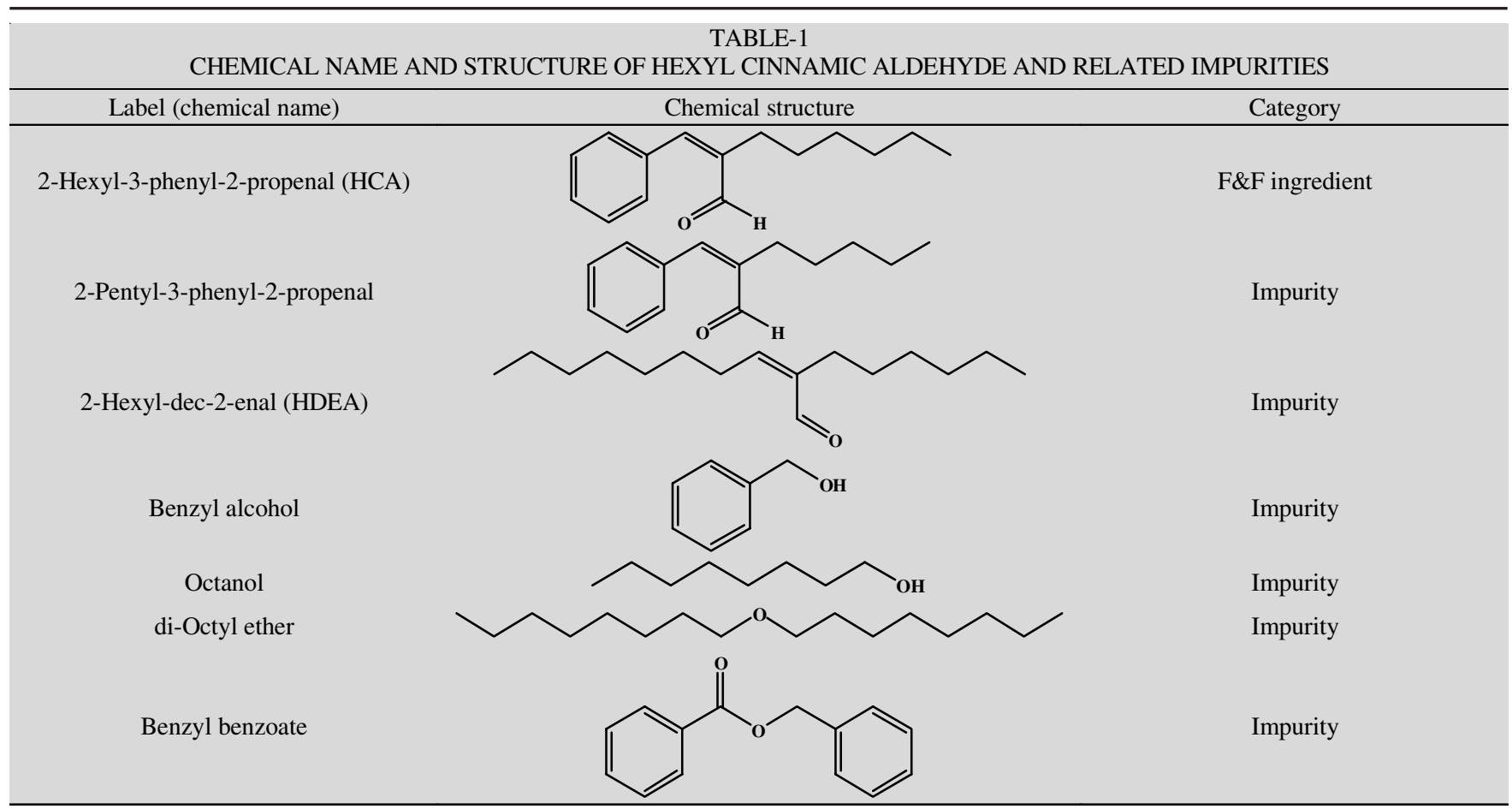

ionization detector and auto liquid sampler were used. All the instruments were calibrated during method development and validation. The isopropyl alcohol is used as solvent for sample preparation. The Agilent make columns having non-polar (HP1) and polar (DB-wax) stationary phases with $30 \mathrm{~m}$ length, $0.25 \mu \mathrm{m}$ internal diameter and $0.25 \mu \mathrm{m}$ thickness were used [9].

\section{RESULTS AND DISCUSSION}

Method development: The gas chromatography is prime analytical technique for separation of volatile organic compound [10]. Its high efficiency allows the separation of component from the mixture in reasonable time. The evolution of stationary phase, capillary column in terms of length, thickness, as well as internal diameter and selection of carrier gas accelerated the development of the newer analytical methods. In order to achieve the separation of the relatively lipophilic 2-hexyl-dec-2-enal (HDEA) impurity from the hexyl cinnamic aldehyde (HCA), the polar columns like DB-wax and inert cap-FFAP are used. Both these column shows similar performance. The details of this Method-B are given in the Table-2 and the resultant chromatogram is displayed in the Fig. 1. The well separated HDEA (4.26 min) and HCA (7.07 min) peaks are observed, however the cis-and trans-isomers of HCA elute together. On the other hand when the non-polar column (HP-1) is used (Method A) these isomers were resolved, however the HDEA is eluted along with the trans-HCA at $8.49 \mathrm{~min}$ (Fig. 2) and for method parameters (Table-2). The variations in the different method parameters like flow rate, temperature, gradient were not able to resolve the HDEA from trans-HCA. The similar results were observed for the other non-polar RTX-5 and HP-5 column.

Thus in order to achieve the separation of HDEA as well as cis- and trans-HCA, the non-polar and polar columns were connected in series thereby leading to the 2D-GC separation. The resulting chromatogram is shown in the Fig. 3, where the

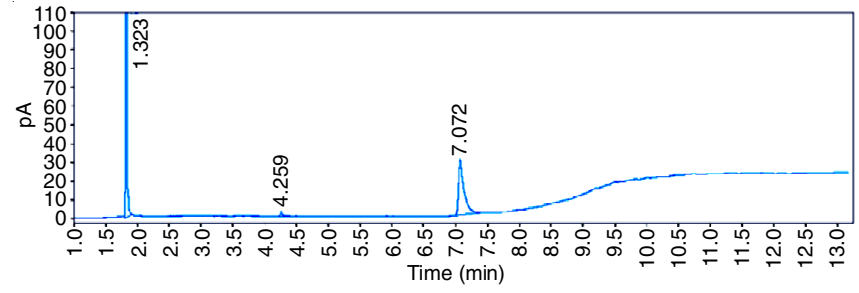

Fig. 1. Chromatogram of GC-FID Method-B with polar column showing HDEA (at $4.259 \mathrm{~min}$ ) is separated from HCA (7.072 $\mathrm{min}$ )

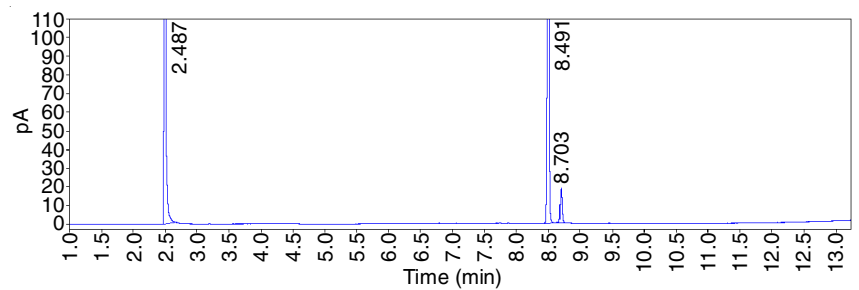

Fig. 2. Chromatogram of GC-FID Method-A with non-polar column showing separation of trans-HCA (8.49 $\mathrm{min})$ and cis-HCA $(8.70 \mathrm{~min})$

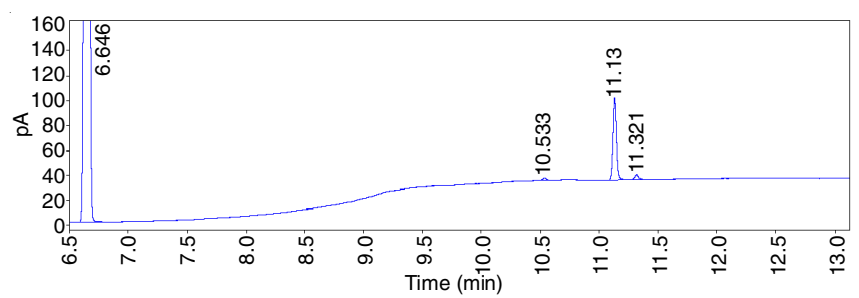

Fig. 3. Chromatogram 2D-GC FID method showing the separation of HDEA (10.53 $\mathrm{min})$ from trans-HCA (11.13 $\mathrm{min})$ and cis-HCA (11.32 $\mathrm{min})$

peak of HDEA (10.53 min), trans-HCA (11.13 min) and cisHCA (11.32 min) are well resolved The corresponding resolution factors for these peaks are 10.80 and 3.50, respectively.

Method validation: The present $2 \mathrm{D}-\mathrm{GC}$ method is validated as per ICH guideline for specificity, selectivity, precision, linearity, accuracy and robustness [11]. 


\begin{tabular}{|c|c|c|c|}
\hline \multicolumn{4}{|c|}{$\begin{array}{c}\text { TABLE-2 } \\
\text { CHROMATOGRAPHIC CONDITIONS FOR 2D GC }\end{array}$} \\
\hline & Method A & Method B & 2D-GC method \\
\hline Instrument & Agilent 7890B with ALS & Agilent 7890B with ALS & Agilent 7890B with ALS \\
\hline Column & $\begin{array}{l}\text { HP-1 }(30 \mathrm{M} \text { length } \times 0.25 \mu \mathrm{m} \\
\text { ID } \times 0.25 \mu \mathrm{m} \text { thickness })\end{array}$ & $\begin{array}{l}\text { DB-wax }(30 \mathrm{M} \text { length } \times 0.25 \\
\mu \mathrm{m} \text { ID } \times 0.25 \mu \mathrm{m} \text { thickness })\end{array}$ & $\begin{array}{l}\text { HP-1 and DB-wax (both } 30 \mathrm{M} \text { length } \\
\times 0.25 \mu \mathrm{m} \text { ID } \times 0.25 \mu \mathrm{m} \text { thickness) }\end{array}$ \\
\hline Detector & FID & FID & FID \\
\hline Flow rate & Constant flow rate: $1.0 \mathrm{~mL} / \mathrm{min}$ & Constant flow rate- $2.5 \mathrm{~mL} / \mathrm{min}$ & Constant flow rate- $2.5 \mathrm{~mL} / \mathrm{min}$ \\
\hline Split ratio & $30: 1$ & $30: 1$ & $30: 1$ \\
\hline Injector and detector temperature & 280 & 280 & 280 \\
\hline Injection volume & $0.1 \mu \mathrm{L}$ & $0.1 \mu \mathrm{L}$ & $0.1 \mu \mathrm{L}$ \\
\hline Column oven temperature & $\begin{array}{l}\text { Initial temperature } 100{ }^{\circ} \mathrm{C} \text {, hold } \\
\text { time } 2 \text { min, gradient of } 30 \\
{ }^{\circ} \mathrm{C} / \mathrm{min} \text { till } 210^{\circ} \mathrm{C} \text {, hold time } \\
5 \mathrm{~min} \text {, gradient of } 20^{\circ} \mathrm{C} / \mathrm{min} \\
\text { till } 260^{\circ} \mathrm{C} \text {, hold time } 0.5 \mathrm{~min}\end{array}$ & $\begin{array}{l}\text { Initial temperature } 160^{\circ} \mathrm{C} \text {, } \\
\text { gradient of } 30^{\circ} \mathrm{C} / \mathrm{min} \text { till } 210 \\
{ }^{\circ} \mathrm{C} \text {, hold time } 5 \mathrm{~min} \text {, gradient of } \\
20^{\circ} \mathrm{C} / \mathrm{min} \text { till } 260^{\circ} \mathrm{C} \text {, hold time } \\
4 \mathrm{~min}\end{array}$ & $\begin{array}{l}\text { Initial temperature } 160^{\circ} \mathrm{C} \text {, gradient } \\
\text { of } 30^{\circ} \mathrm{C} / \text { min till } 210^{\circ} \mathrm{C} \text {, hold time } \\
5 \text { min, gradient of } 20^{\circ} \mathrm{C} / \text { min till } \\
260^{\circ} \mathrm{C} \text {, hold time } 4 \mathrm{~min}\end{array}$ \\
\hline Run time & $13.66 \mathrm{~min}$ & $13.16 \mathrm{~min}$ & $13.16 \mathrm{~min}$ \\
\hline Detector air flow & $300 \mathrm{~mL} / \mathrm{min}$ & $300 \mathrm{~mL} / \mathrm{min}$ & $300 \mathrm{~mL} / \mathrm{min}$ \\
\hline Detector hydrogen flow & $30 \mathrm{~mL} / \mathrm{min}$ & $30 \mathrm{~mL} / \mathrm{min}$ & $30 \mathrm{~mL} / \mathrm{min}$ \\
\hline Carrier & Nitrogen & Nitrogen & Nitrogen \\
\hline
\end{tabular}

Selectivity: The selectivity refers to the extent to which the method can be used to determine particular analyte in mixtures or matrices without interferences from other components of similar behaviour. It simply means that at a given retention time only one component of the sample is being eluted. The selectivity of the method was evaluated by spiking plausible impurities under same experimental condition and distinguishing their retention time from the desired components in test sample [12].

Thus, the elution order of other impurities like octanol, benzyl alcohol, dioctyl ether, 2-pentyl-3-phenyl-2-propenal, benzyl benzoate, HDEA and trans-HCA as well as cis-HCA was studied by spiking these impurities one by one in the isopropyl alcohol and recording their chromatogram. The chromatogram of the spiked impurities in the test solution has been shown in the Fig. 4 and the retention times as well as the other peak factors are given in Table-3. The elution order is as follows: octanol, benzyl alcohol, dioctyl ether, 2-pentyl-3phenyl-2-propenal, HDEA, trans-HCA, cis-HCA and benzyl benzoate. The resolution factor between trans-HCA and cisHCA is 3.50 indicates the good separation of these peaks. The resolution factor for 2-pentyl-3-phenyl-2-propenal and HDEA is 4.49 , rest all the peaks have even larger values of resolution factor. Therefore present 2D-GC method selectively resolve the targeted analytes viz. HDEA, trans-HCA and cis-HCA.

Linearity: Linearity is a capability of method to produce the response towards the analyte concentration in samples.

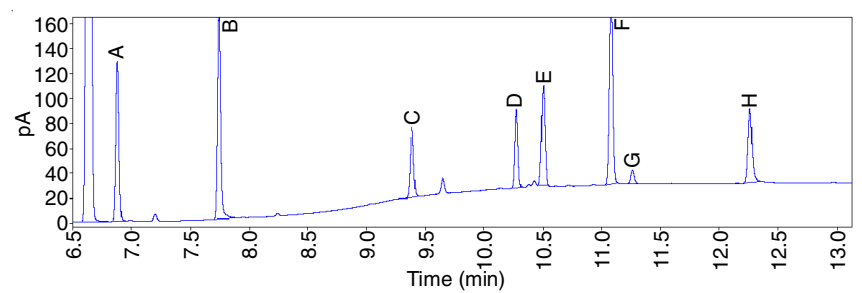

Fig. 4. Spiked chromatogram of all probable impurities for resolution. A: octanol, B: Benzyl alcohol, C: dioctyl ether, D: 2-pentyl-3-phenyl2-propenal, E: HDEA, F: trans-HCA, G: cis-HCA, H: Benzyl benzoate

The method is linear if the response is directly proportional to the concentration of the analyte in the matrix within the range of analyte concentration of interest. The linearity of method is estimated for both HCA and HDEA by recording the chromatograms of their five standards of different concentrations in the desired range. The linearity plots of HDEA, trans-HCA and cisHCA are shown in Fig. 5. The HDEA standards were prepared in concentration range from $10 \mathrm{ppm}$ to $50 \mathrm{ppm}$. The linearity for the detector response towards the concentration of HDEA yield a correlation coefficient of 0.997 . Then the $4000 \mathrm{ppm}$ solution of the HCA is prepared and the amount of HDEA present in it is detected using the aforementioned calibration curve. The concentration of the HDEA calculated from peak area (1.95) is turned out to be $44.4 \mathrm{ppm}$. Thus the amount of HDEA in the original HCA is turned out to be $1.11 \%$, in other words the purity of total HCA is $98.89 \%$. Furthermore from

TABLE-3

RESOLUTION OF OTHER IMPURITIES FROM HDEA, trans-HCA AND cis-HCA

\begin{tabular}{|c|c|c|c|c|c|c|c|}
\hline $\begin{array}{l}\text { Retention } \\
\text { time (min) }\end{array}$ & Peak & Peak name & $\begin{array}{c}\text { Peak } \\
\text { symmetry }\end{array}$ & $\begin{array}{c}\text { Peak } \\
\text { resolution }\end{array}$ & Area & $\begin{array}{c}\text { Peak } \\
\text { selectivity }\end{array}$ & $\begin{array}{c}\text { Peak theoretical } \\
\text { plates }\end{array}$ \\
\hline 6.87 & $\mathrm{~A}$ & Octanol & 0.86 & 4.77 & 231.98 & 1.04 & 335643 \\
\hline 7.74 & B & Benzyl alcohol & 0.78 & 18.24 & 322.60 & 1.14 & 422281 \\
\hline 9.38 & $\mathrm{C}$ & di-Octyl ether & 0.92 & 31.79 & 119.25 & 1.24 & 456672 \\
\hline 10.27 & $\mathrm{D}$ & 2-Pentyl-3-phenyl-2-propenal & 1.00 & 17.38 & 111.57 & 1.10 & 778580 \\
\hline 10.50 & $\mathrm{E}$ & HDEA & 1.15 & 4.49 & 162.08 & 1.02 & 600172 \\
\hline 11.07 & $\mathrm{~F}$ & trans-HCA & 1.05 & 10.80 & 324.45 & 1.06 & 717335 \\
\hline 11.26 & $\mathrm{G}$ & $c i s-\mathrm{HCA}$ & 0.87 & 3.50 & 22.01 & 1.01 & 689343 \\
\hline 12.26 & $\mathrm{H}$ & Benzyl benzoate & 0.88 & 10.62 & 136.59 & 1.09 & 696344 \\
\hline
\end{tabular}

HCA = hexyl cinnamic aldehyde; HEDA = 2-hexyl-dec-2-enal 

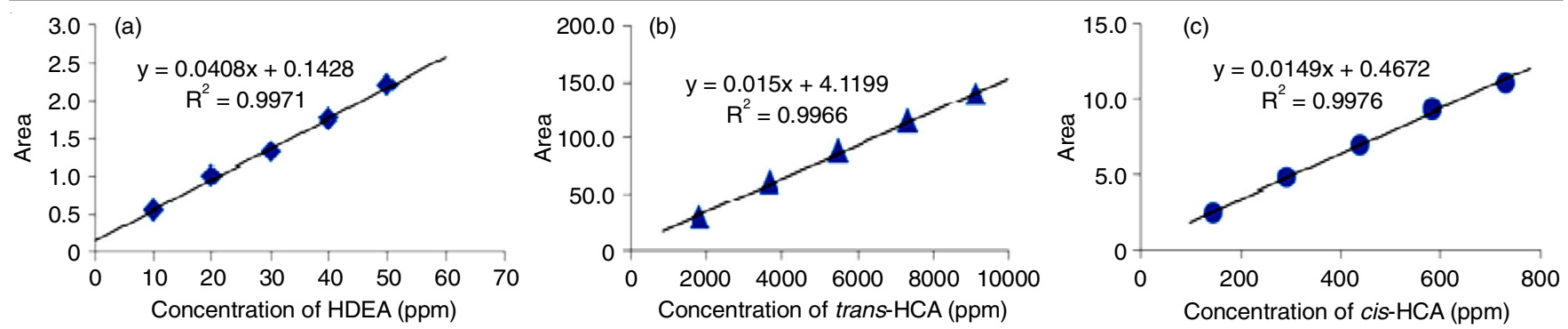

Fig. 5. Linearity graph for HDEA (a), trans-HCA (b) and cis-HCA (c)

the peak areas of the trans-HCA (60.60) and cis-HCA (4.86) their isomer ratio was calculated to be $92.57: 7.43$. The total amount of HDEA, trans-HCA and cis-HCA present in $100 \mathrm{~g}$ of HCA stock is turned out to be $1.11 \mathrm{~g}, 91.56 \mathrm{~g}$ and $7.34 \mathrm{~g}$, respectively. Using $99.89 \%$ purity of $\mathrm{HCA}$, the standard solutions of HCA are prepared to generation the calibration curve of trans-HCA and cis-HCA. Their respective concentration ranges are $1832-9160 \mathrm{ppm}$ and $146-730 \mathrm{ppm}$. The correlation coefficient for linear regression is greater than 0.99 in both these cases. Therefore it is confirmed that the method is liner in the given range.

Precision: Precision provides an indication of agreement among individual test results when an analytical method is used repeatedly to multiple samples. Precision studies are performed when the entire analytical method procedure is finalized. System precision studies carried out on six replicate analysis of standard 10000 ppm HCA sample.

The method precision has been performed by replicate injecting test a sample (of $3000 \mathrm{ppm}$ ) for six times and the intermediate precision is determined by analyzing test sample at different day using same method and column. The data related to the precision study is represented in Table-4. The \% RSD of six replicate HCA standards sample is $1.79,1.58$ and 1.42 for HDEA, trans-HCA and cis HCA respectively. The \% RSD of method precision was observed $1.34,1.21$, and 1.48 , respectively and the corresponding values for intermediate precision were $1.20,1.32$ and 0.70 . The $\%$ RSD of all samples are less than 2.0, hence the method and system said to be precise.

Accuracy: The accuracy study indicates the closeness of the experimental values to the true value. Method accuracy is analyzed by carrying out recovery study of the sample which is known concentration of trans-HCA, cis-HCA and HDEA. The recovery data is represented in Table-4. Results of recovery study of test sample were compared with standard and it is determined using following formula:

$$
\text { Accuracy }=\frac{\text { Area of sample } \times \text { Conc. of stanard }}{\text { Area of standard } \times \text { Conc. of sample }} \times 100
$$

$\%$ RSD for the recovery of the trans-HCA, cis-HCA and HDEA is turned out to be $1.21,1.48$ and 1.34 , respectively. The results obtained are within the limit $(2.0 \%)$ which indicates that the method is accurate.

Limit of detection and limit of quantification: There are several approaches to determine detection and quantification limit i.e. visual evaluation, signal to noise ratio $(\mathrm{S} / \mathrm{N})$ and statistical method based on calibration curve [13]. Here S/N was used to evaluate LOD and LOQ, this was done by serial dilution of HDEA and HCA in isopropyl alcohol till the consistent detectable lower concentration is achieved. The signal of samples with a known amount of low level analyte is compared with the noise of blank sample. From the accepted $\mathrm{S} / \mathrm{N}$ for LOD are 3 and for LOQ is $\geq 10$. The LOD and LOQ values for HDEA are 5.0 and $8.0 \mathrm{ppm}$, respectively and for the HCA are 10.0 and $15.0 \mathrm{ppm}$, respectively.

Robustness: The robustness of analytical method is determined by changing method parameter such as flow rate and column oven temperature. The flow rate was changed \pm 0.5 $\mathrm{mL} / \mathrm{min}$ and the column oven temperature is changed by \pm 5 ${ }^{\circ} \mathrm{C}$ from $160{ }^{\circ} \mathrm{C}$ in original method. The data of the robustness studies are represented in Table-5. When flow rate and temperature is decreased, the retention times were prolonged by 0.75 , 1.18 and $2.21 \mathrm{~min}$, respectively for HDEA, trans-HCA and cis-HCA. On the contrary these retention times for increased flow rate and temperature were shortened by $2.36,2.37$ and $2.43 \mathrm{~min}$, respectively. The \% RSD for robustness studies were observed to be $0.99,1.25$ and 1.24, respectively for HDEA, trans-HCA and cis-HCA, when flow rate and temperature are decreased, whereas for increased flow rate and temperature corresponding \% RSD values are $1.85,1.62$ and 1.74 . There was insignificant change in values of the system suitability

TABLE 4

COMPARATIVE DATA OF PRECISION AND RECOVERY STUDIES OF trans-HCA, cis-HCA AND HDEA

\begin{tabular}{|c|c|c|c|c|c|c|c|c|c|c|c|c|c|c|c|}
\hline \multirow{3}{*}{ 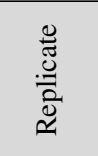 } & \multirow{2}{*}{\multicolumn{3}{|c|}{ Retention time (min) }} & \multicolumn{9}{|c|}{ Area } & \multirow{2}{*}{\multicolumn{3}{|c|}{ Recovery standard (\%) }} \\
\hline & & & & \multicolumn{3}{|c|}{ Standard sample } & \multicolumn{3}{|c|}{ Test sample } & \multicolumn{3}{|c|}{ Test sample (day 2) } & & & \\
\hline & $\begin{array}{c}\text { trans- } \\
\text { HCA }\end{array}$ & $\begin{array}{c}\text { cis- } \\
\text { HCA }\end{array}$ & HDEA & $\begin{array}{c}\text { trans- } \\
\text { HCA }\end{array}$ & $\begin{array}{c}\text { cis- } \\
\text { HCA }\end{array}$ & HDEA & $\begin{array}{c}\text { trans- } \\
\text { HCA }\end{array}$ & $\begin{array}{c}c^{c i s-} \\
\text { HCA }\end{array}$ & HDEA & $\begin{array}{c}\text { trans- } \\
\text { HCA }\end{array}$ & $\begin{array}{c}\text { cis- } \\
\text { HCA }\end{array}$ & HDEA & $\begin{array}{c}\text { trans- } \\
\text { HCA }\end{array}$ & $\begin{array}{c}\text { cis- } \\
\text { HCA }\end{array}$ & HDEA \\
\hline 1 & 11.16 & 11.35 & 10.55 & 138.37 & 11.12 & 5.03 & 40.35 & 3.23 & 1.56 & 40.99 & 3.99 & 1.96 & 97.20 & 96.82 & 103.38 \\
\hline 2 & 11.16 & 11.35 & 10.55 & 142.80 & 11.37 & 5.05 & 40.07 & 3.35 & 1.55 & 41.33 & 3.99 & 1.95 & 96.53 & 100.42 & 102.72 \\
\hline 3 & 11.16 & 11.35 & 10.55 & 143.78 & 11.49 & 5.14 & 40.39 & 3.23 & 1.52 & 40.83 & 3.92 & 1.94 & 97.30 & 96.82 & 100.73 \\
\hline 4 & 11.16 & 11.35 & 10.55 & 141.51 & 11.37 & 5.08 & 41.18 & 3.22 & 1.55 & 41.81 & 3.98 & 1.91 & 99.20 & 96.52 & 102.72 \\
\hline 5 & 11.16 & 11.35 & 10.55 & 140.48 & 11.02 & 4.91 & 41.2 & 3.27 & 1.57 & 41.93 & 3.95 & 1.96 & 99.25 & 98.02 & 104.04 \\
\hline 6 & 11.16 & 11.35 & 10.55 & 143.10 & 11.36 & 4.92 & 41.07 & 3.25 & 1.52 & 42.24 & 3.98 & 1.97 & 98.94 & 97.42 & 100.73 \\
\hline$\%$ RSD & 0.01 & 0.01 & 0.00 & 1.42 & 1.58 & 1.79 & 1.21 & 1.48 & 1.34 & 1.32 & 0.70 & 1.20 & 1.21 & 1.48 & 1.34 \\
\hline
\end{tabular}

$\mathrm{HCA}=$ hexyl cinnamic aldehyde HEDA = 2-hexyl-dec-2-enal 
TABLE-5

ROBUSTNESS STUDY DATA OF trans-HCA, cis-HCA AND HDEA

\begin{tabular}{|c|c|c|c|c|c|c|c|c|c|c|c|c|}
\hline \multirow{3}{*}{ Replicate } & \multicolumn{6}{|c|}{ Set-I: Set-I: Initial temperature $155^{\circ} \mathrm{C}$ and flow 2.0} & \multicolumn{6}{|c|}{ Set-II: Initial temperature $165^{\circ} \mathrm{C}$ and flow 3.0} \\
\hline & \multicolumn{3}{|c|}{ Retention time $(\mathrm{mn})$} & \multicolumn{3}{|c|}{ Area } & \multicolumn{3}{|c|}{ Retention time $(\mathrm{mn})$} & \multicolumn{3}{|c|}{ Area } \\
\hline & HDEA & $\begin{array}{c}\text { trans- } \\
\text { HCA }\end{array}$ & $\begin{array}{c}c i s- \\
\text { HCA }\end{array}$ & HDEA & $\begin{array}{c}\text { trans- } \\
\text { HCA }\end{array}$ & $\begin{array}{c}\text { cis- } \\
\text { HCA }\end{array}$ & HDEA & $\begin{array}{c}\text { trans- } \\
\text { HCA }\end{array}$ & $\begin{array}{c}\text { cis- } \\
\text { HCA }\end{array}$ & HDEA & $\begin{array}{c}\text { trans- } \\
\text { HCA }\end{array}$ & $\begin{array}{c}\text { cis- } \\
\text { HCA }\end{array}$ \\
\hline 1 & 11.91 & 12.53 & 12.76 & 1.03 & 36.02 & 1.89 & 9.55 & 10.16 & 10.33 & 1.16 & 39.61 & 3.61 \\
\hline 2 & 11.91 & 12.53 & 12.76 & 1.00 & 36.63 & 1.85 & 9.55 & 10.16 & 10.32 & 1.16 & 39.62 & 3.66 \\
\hline 3 & 11.91 & 12.53 & 12.76 & 1.00 & 36.47 & 1.89 & 9.55 & 10.16 & 10.33 & 1.20 & 39.82 & 3.62 \\
\hline 4 & 11.90 & 12.53 & 12.75 & 1.01 & 36.43 & 1.84 & 9.55 & 10.16 & 10.32 & 1.15 & 38.10 & 3.59 \\
\hline 5 & 11.90 & 12.53 & 12.76 & 1.00 & 37.27 & 1.85 & 9.56 & 10.16 & 10.32 & 1.13 & 38.92 & 3.48 \\
\hline 6 & 11.91 & 12.53 & 12.76 & 1.01 & 36.06 & 1.85 & 9.56 & 10.16 & 10.32 & 1.16 & 39.39 & 3.65 \\
\hline SD & 0.004 & 0.001 & 0.004 & 0.01 & 0.46 & 0.02 & 0.002 & 0.000 & 0.001 & 0.02 & 0.64 & 0.06 \\
\hline$\%$ RSD & 0.030 & 0.010 & 0.034 & 0.99 & 1.25 & 1.24 & 0.022 & 0.04 & 0.013 & 1.85 & 1.62 & 1.74 \\
\hline
\end{tabular}

criteria like tailing factor, theoretical plates and \% RSD. The values of these studies are well within acceptance limit.

\section{Conclusion}

Accurate, linear, specific, robust, and precise 2D GC method is developed and is successfully validated as per the criteria of ICH guideline for analytical method validation for the quantitative analysis of flavour and fragrance ingredient hexyl cinnamic aldehyde and its impurity 2-hexyl-dec-2-enal. The HDEA peak is well resolved from HCA and its other known impurities. The method is linear with correlation coefficient being greater than 0.99 , and system, method and intermediate precision are evaluated and \% RSD was found within limit. Robustness also does not show significant change for the system suitability. The present 2 dimensional gas chromatographic method is useful for routine quantitative analysis of both the cis- and trans- isomers of HCA as well as its major impurity HDEA.

\section{ACKNOWLEDGEMENTS}

The authors thank G.T. Nikam, Eternis Fine Chemicals Ltd., Pune, India for his constant support and encouragment. One of the authors (RVP) thank Swami Ramanand Teerth Marathwada University, Nanded, India for the MRP (APDS/ Uni.MRP-IV/Sci.\&Tech-Chem.Sci./2017-18/2986).

\section{REFERENCES}

1. T. Rustemeyer, P. Elsner, S.M. John and H.I Maibach, Kanerva's Occupational Dermatology, Aromatherapist, 118, 1266 (2012).

2. (a) G. Shi-long, China Surf. Det. Cosm. Syn., 1, 17 (2006); (b) Z. Lirong and Y. Feng-ping, Chem. World, 12, 10 (2014).

3. International Fragrance Association (IFRA); $\alpha$-Hexyl Cinnamic aldehyde, 47th amendment (2013).

4. S.M. Cohen, S. Fukushima, N.J. Gooderham, S.S. Hecht, L.J. Marnett, I.M.C.M. Rietjens, R.L. Smith, M. Bastaki, M.M. Mcgowen, C. Harman and S.V. Taylor, 27th publication by the Expert Panel of Flavor and Extract Manufacturers. Association (FEMA), GRAS 27, Food Technology (2011)

5. D. Rowe, Chemistry and Technology of Flavor and Fragrances, WileyBlackwell Publishing Ltd, UK, edn 1, p. 351 (2009).

6. Sigma-Aldhrich, GC Column Selection Guide, Achieve Optimal Method Performance, Nicholas, Walter, p. 22.

7. C. Vendeuvre, R. Ruiz-Guerrero, F. Bertoncini, L. Duval and D. Thiébaut, Oil Gas Sci. Technol., 62, 43 (2007);

https://doi.org/10.2516/ogst:2007004

8. V. Ravichandra, S. Shalini, K.M. Sundram and R. Harish, Int. J. Pharm. Pharm. Sci., 2(Suppl 3), 18 (2010).

9. Column Selection Principles, Agilent J\&W GC Column Selection Guide, pp. 19.

10. A.G. Piantanida and A. R. Barron, Principles of Gas Chromatography, OpenStax-CNX, (1.2) pp. 1-12 (2014).

11. ICH Expert, ICH Harmonized Tripartite Guideline, Validation of Analytical Test Procedures and Method, Q2(R1), pp. 17 (2005).

12. J. Vessman, R.I. Stefan, J.F. van Staden, K. Danzer, W. Lindner, D.T. Burns, A. Fajgelj and H. Müller, Pure Appl. Chem., 73, 1381 (2001); https://doi.org/10.1351/pac200173081381.

13. S.B. Puranik, V.R. Pawar, N. Lalitha, P.N.S. Pai and G.K. Rao, Orient. J. Chem., 24, 529 (2008). 